\title{
Interet du dosage de la prolactinemie dans le bilan d'une insuffisance erectile. A propos de 341 Patients
}

\author{
D.DelavierRe, PH.GIRARD, H.Ibrahim, M.PENEAU
}

Service d'Urologie-Andrologie, CHR d'ORLEANS

\section{INTRODUCTION}

Environ $90 \%$ des hyperprolactinémies (HPRL) importantes s'accompagnent de troubles sexuels, notamment baisse de la libido et insuffisance érectile. En conséquence le dosage de la prolactinémie a été proposé dans le bilan de l'insuffisance érectile pour rechercher une HPRL et une endocrinopathie sousjacente. Mais ce dosage est il vraiment justifié ?

\section{MATERIEL ET METHODES}

Du 1er/09/1992 au 31/08/1996, 401 patients ont consulté en raison d'une insuffisance érectile.

341 (âge moyen $=52,2$ ans) ont bénéficié d'un ou plusieurs dosages de la prolactinémie (PRL).

Parmi ces 341 patients $95(27,8 \%)$ présentaient une baisse de la libido associée à l'insuffisance érectile.

Ce dosage n'a pas été réalisé chez 60 patients (35 étiologies organiques non hormonales évidentes, 15 non respects de la prescription, 10 sans raison précise).

\section{RESULTATS}

18 patients avaient une hyperprolactinémie (HPRL)> $25 \mathrm{ng} / \mathrm{ml} .12$ contrôles ont été effectués et 11 se sont révélés normaux. I1 reste donc 7 HPRL $(2,1 \%)$ dont 6 non contrôlées par un second dosage : 4 HPRL ont une origine médicamenteuse (Sulpiride ou Amisulpride) et trois une origine imprécise (PRL $<35$ $\mathrm{ng} / \mathrm{ml}$, pas de contrôle ni bilan car patients perdus de vue ou n'ayant pas respecté la pres- cription de contrôle). Les 7 patients ont une testostéronémie normale. $2(28,6 \%)$ ont une libido émoussée.

\section{REVUE DE LA LITTERATURE}

L'analyse de 11 séries récentes de la littérature montre que $3,5 \%(1,4$ à $7,6 \%)$ des insuffisants érectiles étudiés (souvent sélectionnés selon des critères différents) ont une PRL > $15 \mathrm{~g} 25 \mathrm{ng} / \mathrm{ml}$ mais seulement $1,3 \%$ une PRL > 35 ou $40 \mathrm{ng} / \mathrm{ml}$. 0,6\% (O à $3,8 \%$ ) ont une tumeur hypophysaire. Les HPRL secondaires à une tumeur hypophysaire sont souvent élevées $(<30 \mathrm{ng} / \mathrm{ml}$ dans $90 \%$ des cas) et s'accompagnent habituellement d'une libido émoussée (80 à 100\% des cas) et d'une hypotestostéronémie $<3 \mathrm{ng} / \mathrm{ml}$ ( $90 \%$ des cas). Seules les HPRL $>35$ ou $40 \mathrm{ng} / \mathrm{ml}$ peuvent expliquer une insuffisance érectile.

\section{CONCLUSIONS}

1. La PRL doit être prélevée dans des conditions précises et contrôlée en cas d'élévation.

2. La prévalence des HPRL, et des tumeurs hypophysaires dans la population des insuffisants érectiles est faible.

3. Le dosage de la PRL est justifié si la libido est émoussée, la testostéronémie totale $<3$ $\mathrm{ng} / \mathrm{ml}$ ou si le patient présente des signes tels que gynécomastie, troubles visuels ou céphalées.

Ce texte a été publié dans le numéro précédent d'Andrologie (p.99) mais amputé de la Revue de la Littérature et des conclusions. Nous en rétablissons ici la version complète. 